Vol. 13, n 1 | 2009

Varia

\title{
La dimension doctrinale du procès dans l'histoire de la justice criminelle : La leçon historiographique de Mario Sbriccoli
}

Massimo Meccarelli

\section{(2) OpenEdition Journals}

Édition électronique

URL : https://journals.openedition.org/chs/693

DOI : $10.4000 /$ chs.693

ISSN : 1663-4837

Éditeur

Librairie Droz

\section{Édition imprimée}

Date de publication : 1 mars 2009

Pagination : 73-89

ISBN : 978-2-600-01295-9

ISSN : 1422-0857

\section{Référence électronique}

Massimo Meccarelli, «La dimension doctrinale du procès dans l'histoire de la justice criminelle : La leçon historiographique de Mario Sbriccoli », Crime, Histoire \& Sociétés / Crime, History \& Societies [En ligne], Vol. 13, $n^{\circ} 1$ | 2009, mis en ligne le 01 mars 2012, consulté le 25 mars 2022. URL : http:// journals.openedition.org/chs/693 ; DOI : https://doi.org/10.4000/chs.693 


\title{
La dimension doctrinale du procès dans l'histoire de la justice criminelle
}

\author{
La leçon historiographique de Mario Sbriccoli*
}

\author{
Massimo Meccarelli
}

Je ne parlerai pas des cultures qui se perdent. La constance de la vie, la continuité durable de la vie, est une certitude de l'art.

(J. L. Borges, Inquisiciones)

The aim of this essay is to outline the historiographical theory of Mario Sbriccoli with particular reference to his analysis of the criminal process in the late medieval and early modern period. A central issue here is the way in which he integrated different disciplines interested in the criminal problem, and used them an opportunity for exploring the history of criminal justice. His main conclusions - the discovery of a pluralistic dimension of justice, the variability of the process structure, the particular contribution of juridical science, in correspondence with common practice, in the construction of the penal system) are analysed. Finally, the historiographical perspectives are considered, which thanks to this methodological approach and these results, open additional areas for research.

Cet essai a pour but de présenter la théorie historiographique de Mario Sbriccoli et plus particulièrement son analyse du processus pénal au MoyenÂge tardif et au début de l'époque moderne. L'aspect central est la manière dont ce dernier a intégré différentes disciplines concernées par le problème du crime et les a utilisées pour explorer l'histoire de la justice pénale. Ses principales conclusions sont analysées ici: la découverte de la dimension plurielle de la justice; la variabilité de la structure du processus; la contribution spécifique de la science juridique à la construction du système pénal, en relation avec la pratique commune. L'article examine enfin les perspectives historiographiques et leur ouverture à de nouveaux domaines de recherche, à partir de cette démarche méthodologique et de ses résultats.

$\mathrm{T}$ rouver les mots pour recueillir les idées; réfléchir sur la leçon historiographique de Mario Sbriccoli, c'est aussi l'occasion de se réapproprier un lien intellectuel qui, après son décès et avec le sentiment persistant de son absence, reste fort et vital. Il s'agit d'évoquer un itinéraire scientifique aussi unique que capable de proposer des idées très actuelles et des clés d'interprétation de l'expérience

* Nous proposons ici, en langue française et avec des modifications marginales, l'essai en cours de publication in Madero, Conte. Mise au point du texte: B. Leconte, R. Lévy, M. Porret. 
juridique ${ }^{1}$. Exercice nécessaire, qui ne peut être renvoyé à plus tard, bien qu'empreint de la nette sensation d'inadéquation de l'élève qui tente de se mesurer au talent de son maître.

Je tenterai d'abord de retrouver dans son œuvre sa méthode, ses instruments, ses schémas analytiques, ses catégories d'interprétation; puis j'analyserai les résultats qu'il a obtenus et réfléchirai aux perspectives historiographiques que l'œuvre de Mario Sbriccoli nous offre.

\section{L'INTÉGRATION DES SAVOIRS}

Mario Sbriccoli n'a pas pu terminer l'Histoire du pénal qu'il se proposait d'écrire depuis des années; celle-ci est pourtant déjà présente de façon accomplie dans ses recherches. Son itinéraire d'historien s'est en effet déroulé pas à pas, à travers des vérifications attentives, des pondérations et des revirements, d'où a surgi un parcours historiographique formidable qui se présente à nous, aujourd'hui, cohérent, organique et unitaire. La méthode qu'il a choisie a été décisive à ce propos; elle a en effet eu les caractéristiques de la fondation ex ante; c'est-à-dire qu'elle a permis d'établir le domaine d'enquête, d'en choisir les approches et d'en élaborer les instruments.

Cet ancrage méthodologique prend sa source dans l'idée de mener une enquête scientifique sur le droit qui prenne également en compte la complexité dans laquelle l'élément juridique est plongé. Ce choix de terrain est déjà celui de son premier livre $^{2}$, où le thème de l'interprétation du statut médiéval se transforme en une réflexion sur la fonction des juristes à l'ère des communes ${ }^{3}$. Appliquée à la matière du pénal que Mario Sbriccoli ne quittera plus tout au long de ses travaux, cette position a pris par la suite des contours encore plus définis. Essayons de les relire.

Dans les années 1970, l'historien du droit pénal semblait appelé à jouer un triple rôle ${ }^{4}$ : indiquer «ce que tout système produit de politique»; étudier systématiquement «la réflexion menée sur le lien entre droit et politique $»^{5}$; considérer le droit pénal dans «sa centralité dans l'histoire et dans la lutte civile», c'est-à-dire, non seulement en tant qu'instrument de pouvoir mais aussi en tant que lieu de la confrontation politique et sociale, soit «le terrain même sur lequel on revendique et conteste les droits, les privilèges et les places».

Pour accomplir ce programme inédit, il était nécessaire de trouver le dimensionnement épistémologique approprié: celui-ci s'est constitué à partir du constat de la forte relation qui existait entre le «moment juridique» et la «conscience de la société» ainsi que «son fonctionnement» ${ }^{6}$. Les caractères du pénal, selon la leçon

1 Voir par exemple les résultats du Colloque qui lui a été consacré (Macerata 26-28 octobre 2006) dans le volume Lacchè, Latini, Marchetti, Meccarelli (2007).

2 Sbriccoli (1969).

3 Voir à ce propos Grossi (2004-2005, p. 1393); Lacchè (2005, p. 403sqq.); Cappellini (2007, p. 89). Voir Sbriccoli (1969, p. 3; 1972, pp. 99-101; 1974, p. 3; 1976, pp. 205-210; 1997a, pp. 37-38; 2007, pp. 139-148).

$4 \quad$ Sbriccoli (1976, pp. 207-208).

5 Et aussi, «sur le rapport entre intérêts économiques des classes dominantes ou émergentes, leurs stratégies politiques et leur instrument pénal» Sbriccoli (1976, pp. 207-208).

6 Sbriccoli (1986a, p. 130). Cfr. Grossi (2004-2005, pp. 1395-1396); Caroni, (2005, pp. 27-55). Les thèses exprimées dans cet essai de 1986 ont été par la suite confirmées par Sbriccoli (1991a, p. 89). 
répétée de Mario Sbriccoli, dépendent directement de la constitution matérielle d'une société, parce que le pénal «a naturellement tendance à se conformer aux logiques constitutionnelles des pouvoirs qui l'expriment ${ }^{7}$.

En conséquence, l'histoire du droit est forcément considérée en tant qu'histoire d'une science de la société, bien reliée aux autres sciences sociales ${ }^{8}$. C'est par là que se justifie le souffle interdisciplinaire qui constitue le caractère typique et constant des travaux de Mario Sbriccoli.

Sur le plan programmatique, il faut rechercher et promouvoir un dialogue capable de créer un rapport d'instrumentalité réciproque entre les différentes disciplines historiques (mais pas uniquement celles-ci) concernées par la question pénale 9 . Mario Sbriccoli entend ainsi affirmer un principe d'intégration des savoirs. Il s'agit moins d'un simple appel au dialogue interdisciplinaire, qu'une réelle refondation: il faut se dé-discipliner et acquérir des compétences à l'extérieur de sa discipline $^{10}$. Sur le plan historiographique, observait-il plus récemment, il faut soumettre le thème du pénal à «un ensemble de réactifs disciplinaires », capables de «le compliquer, en le fécondant et en l'éparpillant sur des terrains différents de celui sur lequel, disciplinairement [il aurait dû] être $»^{11}$.

Il ne s'agit évidemment pas de renoncer aux méthodes et approches propres à chacun; chaque spécialiste continuerait, dans cette convergence méthodologique, à mener ses propres recherches selon la spécificité de sa discipline. Toutefois, si les objets d'intérêt spécifiques restent distincts, l'intégration des savoirs devient en quelque sorte (nécessairement) fonctionnelle pour leur travail ${ }^{12}$.

En adoptant une méthode intégrée, Mario Sbriccoli reste donc un historien du droit, c'est-à-dire un juriste qui, en récupérant le sens de certaines paraboles de l'expérience historique, s'insère avec pertinence dans le débat contemporain entre juristes. Mario Sbriccoli a pu ainsi «instaurer un rapport de soutien réciproque - un véritable feed-back - entre l'histoire du phénomène juridique et le mouvement de réforme juridique et institutionnel dont on sent l'urgence et que l'on tente d'amor-

Sbriccoli (2002, p. 167). Voir aussi Sbriccoli (2001, p. 363; 1997b, p. 697).

8 C'est dans ce sens qu'il s'est exprimé dans l'exposé, encore inédit, Le droit à la Renaissance, fait à la Sorbonne, le 9 octobre 2004.

$9 \quad$ Il s'agissait de rassembler des historiens sociaux, des historiens de la criminalité, des historiens des institutions, des historiens de l'expérience religieuse, des historiens de la politique, des historiens de l'économie, des historiens du droit, des juristes, des anthropologues, des sociologues, des philosophes, pour mentionner les principales branches des sciences sociales concernées. Voir à ce propos Sbriccoli (1986a, p. 127; 2001, p. 352). Poussé par cette prise de conscience, Mario Sbriccoli manifeste son appréciation pour l'ouverture interdisciplinaire qui caractérisait le projet éditorial des Materiali de Giovanni Tarello (se référant aussi bien aux terrains de recherche qu'aux instruments utilisés matériellement), qui choisissait comme point de vue l'histoire de la culture juridique. Voir Sbriccoli (1976, p. 206).

10 Comme il le dit dans Sbriccoli (1986a, pp. 137-148). C'est-à-dire qu'il faut acquérir, explique-t-il, «une double compétence d'historiens juristes et sociaux» [p. 148]; c'est un raisonnement qu'il fait aussi dans Repliche, in Grossi (1986, p. 93); voir aussi Sbriccoli (1991a, p. 93).

11 Sbriccoli (2001, p. 352).

12 Il l'affirme clairement in Sbriccoli (1986a, p. 137). Grossi le met également en évidence (2004-2005, pp. 1395-1396); et aussi (2007, p. 455), où il rappelle que cela répondait au but de «fonder une autonomie non artificielle de l'histoire du droit». Du reste la notion de justice criminelle assumée par Mario Sbriccoli est telle qu'elle peut (ou doit) être considérée de plusieurs points de vue; cf. infra note 23 . 
cer dans le pays $\gg^{13}$. Ce n'est pas par hasard s'il a poussé sa recherche au delà de la période médiévale et moderne, en suivant, jusqu'à l'époque contemporaine, ce qu'il a appelé le processus de civilisation du droit pénal ${ }^{14}$.

Mario Sbriccoli a montré la nécessité de conserver l'aspect technico-juridique dans l'identification de l'objet d'étude car «l'histoire du pénal», devait «régler ses comptes avec le moment strictement juridique $»^{15}$. Dans la perspective de l'intégration des savoirs, cette spécificité était également fonctionnelle pour les études d'histoire sociale. C'est en vertu de cette propension, propre au phénomène juridique, à refléter la réalité et, par ailleurs, à enregistrer des signaux et des messages, et grâce à elle, que l'histoire sociale pouvait découvrir des «phénomènes sociaux qui seraient, sinon, asymptomatiques ${ }^{16}$.

Cette position méthodologique l'a amené à repérer un domaine de recherche inédit: celui de l'histoire du crime et de la justice criminelle, vue comme un terrain de rencontre, ou encore comme point de fusion, d'objets et de méthodologies qui appartiennent de façon équilibrée à l'histoire du droit et à l'histoire sociale ${ }^{17}$. L'histoire de la justice criminelle recoupe en même temps «l'histoire de la société et celle des productions normatives des pouvoirs publics, les approches politiques et anthropologiques, les rapports entre justice et pouvoir, les justices plurielles et leur progressive reductio ad unitatem, les cultures et les usages, les mentalités et les pratiques $»^{18}$.

13 Sbriccoli, (1976, p. 206). Ce n'est pas seulement une sensibilité qui émane du climat spécial d'une période, celle des années soixante-dix, dans laquelle le discours sur la militance civile des intellectuels a une importance particulière. Dans un autre contexte et une autre phase, Sbriccoli (1991b, p. 377) percevait de nouveau l'importance de la méthode de l'intégration pour la réflexion scientifique contemporaine. Il est significatif de voir Sbriccoli (1997b, p. 697) s'arrêter sur l'idée de Francisco Tomas y Valiente d'une «connexión entre l'histoire que l'on vit et l'histoire que l'on écrit », comme manière «de participer à l'analyse et donc à la connaissance de la realidad como problema». Lacchè (2005, pp. 406-407) a prouvé combien est importante chez Sbriccoli - dans ses relations scientifiques en Italie (en particulier avec Giovanni Tarello et Franco Bricola) mais aussi au niveau international (avec le Groupe Européen de Recherche sur les Normativités ou l'International Association for the History of Crime and Criminal Justice, etc.) - la rencontre entre la culture juridique et le pénal pour une redécouverte du «rapport naturel et symbiotique entre dimension juridique et historicité de l'expérience ». C'est un point qui renvoie à une idée de juriste politisé, mise en évidence dans l'essai de Cappellini (2007, p. 68sqq.).

14 Sbriccoli (2001, p. 354): par «processus de civilisation» du pénal l'on entend «l'interminable parcours pour 'en terminer avec la vengeance' et la toujours précaire 'arrivée de la garantie'»; voir Sbriccoli (1998, p. 254). Les développements du pénal au cours des siècles sont lus sur la base d'une vision historique originale; Costa (2007, pp. 53-57), en a récemment reconstruit les traits: en considérant l'histoire en tant que praxis, Mario Sbriccoli veut saisir l'implication réciproque du présent et du passé en vue d'effectuer une problématisation de ce rapport; pour déterminer la direction du procès historique, ce dernier est «démonté dans ses phases multiples» et libéré de préjuges herméneutiques [p. 55]. Par cette approche, explique encore Costa, l'on n'attribue «aucune valeur providentialiste et téléologique au procès historique» et encore moins le représente-t-on comme un continuum; le sens de la variété des expériences reste fort, ainsi que la distance de toute «projection acritique du présent dans le passé» [p. 54].

15 Sbriccoli (1997b, p. 700).

16 Sbriccoli (1986a, pp. 141-143; 2001, p. 347; 1988, pp. 499-500; 1991a, p. 90). Cf. Lacchè (2005, p. 409).

17 Sbriccoli (1991a, pp. 89-93; 1988, pp. 489-501).

18 Sbriccoli (2001, p. 354). Voir aussi Sbriccoli (1986a, p. 146; 1988, p. 494; 1991a, p. 91). 
L'histoire intégrée du droit pénal devient ainsi, dans une perspective d'histoire de la justice criminelle, l'histoire du pénal. Ici, le droit est observé comme une partie d'une «expérience juridique d'ensemble» ${ }^{19}$, et considéré dans ses connexions avec la société; et en ce sens - je continue d'utiliser les expressions de Mario Sbriccoli - l'histoire du pénal est «l'histoire d'une pluralité articulée», capable de considérer la matière pénale comme un objet complexe dans ses dimensions normatives. On y retrouve, par exemple, des éléments du droit privé, on y découvre certains caractères du droit public qui ne possèdent pas de connotations étatiques; le procès (dans son acception moderne) parait encore inséré dans un contexte de formes de justice diversifiées ${ }^{20}$.

En outre, l'histoire du pénal permet de découvrir que ces éléments de pluralité ne se situent pas dans une succession évolutive où l'un remplace l'autre selon une séquence logique. Ces éléments «coexistent, se conditionnent et s'influencent». L'histoire du pénal permet de voir cette complexité en tant que système et d'en reconstruire les régimes de fonctionnement interne ${ }^{21}$.

\section{CULTURE DE LA JUSTICE}

Évoquons maintenant les aboutissements des recherches menées sur ces bases méthodologiques, en partant de celle qui sont relatives à la représentation de la dimension de la justice entre la fin du Moyen-Âge et l'époque moderne.

Celle-ci - observe Mario Sbriccoli dans un bilan historiographique des dernières décennies - a été redécouverte en tant qu'objet unitaire mais pluriel, au sens où «elle est unique [...] mais se manifeste et s'affirme selon des modes diversifiés $»^{22}$. La multiplicité des points de vue impliqués dans la recherche sur la justice criminelle met en perspective cette notion de justice dans la complexité qui la caractérise effectivement ${ }^{23}$.

Mario Sbriccoli a voulu réaliser cette reconstruction de la justice dans sa dimension sociale et culturelle complexe sans recourir au paradigme de l'État qui aurait comme effet un certain conditionnement; celui-ci est pertinent pour qualifier surtout la justice pénale au $\mathrm{XIX}^{\mathrm{e}}$ siècle, et dont la logique circonscrit la notion de justice,

\footnotetext{
19 Sbriccoli (1991a, p. 88). Cf. Lacchè (2007, pp. 13-16).

20 Sbriccoli (1993, p. 129). Cf. infra note 31.

21 Sbriccoli (1993, p. 130).

22 Sbriccoli (2001, p. 355). Voir aussi Sbriccoli (1993, p. 129).

23 Sbriccoli (2001,p. 352). Il précisait que les points de vue par lesquels 1'historien du pénal peut considérer la justice sont multiples: «une ressource d'utilisation pour les individus et les communautés dans ces sociétés conflictuelles [...] un moyen d'affirmer une conception du pouvoir qui deviendra progressivement une idée de l'État [...] un des niveaux où se manifeste le conflit politique, et aussi un moyen de le gérer [...] une révélation de la donnée de fond des sociétés médiévales et modernes, lues à travers le paradigme du conflit [...] un instrument d'assurance de l'ordre imposé d'en haut, mais aussi le lieu de la satisfaction personnelle, de la vengeance indirecte [...] indicateur du caractère négociateur et concerté de la société de base communautaire et de classe, mais aussi du processus incessant et inexorable d'une hégémonie des pouvoirs publics [...] le lieu où [...] saisir la coexistence entre règles communautaires 'locales' et normes 'centrales', la dialectique entre droit et pratiques, entre répression et négociations [...] et enfin le glissement progressif [...] vers l'affirmation d'un corps normatif unique, combinaison de lois et jurisprudence qui seulement après la fracture révolutionnaire parvient à effacer impérieusement coutumes et pratiques » (pp. 352-353).
} 
pour la concevoir, sur la base d'un objectif: la défense de la loi et l'application d'une peine à tout infracteur ${ }^{24}$.

L'inadéquation du paradigme de l'État résultait à l'évidence des données que les recherches d'histoire de la justice criminelle faisaient justement émerger. Le problème concernait la compréhension d'une forme de justice communautaire qui se présentait toujours plus comme typique de ces siècles-là (tant au plan quantitatif que qualitatif ${ }^{25}$ ). L'emploi (conscient et inconscient) du paradigme de l'Etat pour l'interpréter menait à des résultats paradoxaux et trompeurs ${ }^{26}$, parce qu'il finissait par placer à l'extérieur de la dimension de la justice quelque chose qui en constituait au contraire la marque caractéristique. Sbriccoli indique à titre d'exemple de ce vice de formulation, certaines catégories interprétatives comme le prétendu infra-judiciaire ou extra-judiciaire ${ }^{27}$.

Le critère de délimitation de cette dimension de la justice unitaire-plurielle ne pouvait pas, du reste, être celui de son objectif, puisque les objectifs de cette justice étaient multiples: "'rendre raison' ou 'donner satisfaction', redresser un tort, réparer un dommage, compenser, indemniser, réconcilier, ou encore infliger une peine, ou plus simplement donner suite à une prévision normative au moyen d'un procès réglé ${ }^{28}$. Le critère de délimitation devient alors, de façon plus ample, le caractère rituel de la justice, c'est-à-dire le fait que la justice soit vue comme «le résultat accepté d'un rite $»^{29}$. Le discours sur la justice finit donc par se concentrer sur le rite, comme lieu juridique dans lequel on observe l'émergence de la dimension pénale publique hors « du complexe indistinct de la conflictualité urbaine confié à la justice négociée et aux transactions $»^{30}$.

Dans cette perspective, les formes dans lesquelles cette justice se manifeste, sont aussi bien celles qui présentent des caractères publics - en ce sens qu'elles répondent à la sauvegarde des intérêts collectifs - bien que ne possédant pas les traits du droit public étatique ${ }^{31}$, que celles dont la vocation est plus typiquement privée.

Sur la base de ces résultats, Mario Sbriccoli parvient à une rationalisation de catégories qui permet de considérer, de façon très efficace, cette justice plurielle

24 Sbriccoli (2001, p. 355).

25 Au Moyen-Âge ainsi qu'à l'époque moderne, non seulement ce type de justice communautaire est 'quantitativement' le plus répandu au niveau de justice pratiquée; il est probablement reconnu par cette société comme le moyen principal de faire justice. Cf. Ibidem, pp. 350-359. Ce fait est aussi confirmé par la résistance opposée à l'application d'importantes législations de l'époque moderne porteuses de la nouvelle justice hégémonique; Sbriccoli (2003, pp. 79-88) prend l'exemple de la Constitutio Criminalis Carolina.

26 Sbriccoli (2001,p. 355). Ce paradigme paraît de manière problématique «enkysté dans l'ordre mental de l'historien du droit, élevé dans un horizon dans lequel les 'conditions de pensabilité' sont englouties dans l'orbite du modèle dualiste État-société» (pp. 348-349). Cf. Sbriccoli (1993, pp. 130-131).

27 Sbriccoli (2001, p. 350; 2007).

28 Sbriccoli (2001, p. 356). Voir aussi Sbriccoli (1993, p. 129).

29 Sbriccoli (2001, p. 355).

30 Sbriccoli (2004, p. 104). Voir aussi Sbriccoli (1998, pp. 259-268).

31 Sbriccoli (2001, p. 357). Les pouvoirs 'publics' sont, dans une réalité dont l'organisation n'est pas connotée par le dualisme État-société, l'«expression d'équilibres communautaires et non pas à la manière de délégués d'une forme étatique qui se superpose à ces équilibres». Ceux-ci sont donc appelés à jouer un rôle de médiation et de recomposition des conflits qui est fonctionnel pour la paix de la communauté. Ce rôle légitime essentiellement, au moins dans la période de la fin du MoyenÂge, le caractère public du pouvoir. Cf. supra note 20 . 
avec ses valeurs historiques unitaires. Je me réfère ici à l'heureuse distinction entre la justice négociée et la justice hégémonique ${ }^{32}$.

La première lui a servi à indiquer la justice communautaire de la médiation, qui tend à se substituer à la vengeance, en obtenant, en même temps que la satisfaction de la victime, une composition du litige. C'est la justice qui appelle dans la cause les entourages respectifs qui interviennent à différents niveaux dans le processus de négociation, et qui comprend les différentes figures de justice: celle «obtenue par la simple négociation entre les intéressés à l'exclusion d'une quelconque formalité, celles qui impliquent une médiation sociale, ainsi que celles qui tirent parti de la structure judiciaire publique, si cette dernière se laisse impliquer dans la négociation en s'en faisant la promotrice, fiduciaire ou garante». Ce type de justice «est basé davantage sur le consensus que sur la certitude $»^{33}$.

Par contre, par justice hégémonique, il a évoqué une justice qui veut affirmer et promouvoir le principe de l'obéissance à la loi, plutôt que d'obtenir un accommodement du conflit. Elle déplace le sens de la notion de la justice elle-même vers la sphère sémantique «de la répression et de la 'lutte contre le crime', et la retire de celle de la justice distributive et réparatrice ${ }^{34}$. Contrairement à la précédente, ce type de justice est basé «sur la certitude et non nécessairement sur le consensus » ${ }^{35}$. C'est, en effet, la connotation hégémonique qui tend à réduire la justice à l'application pure et simple de la loi.

Dans la reconstruction qu'en fait Sbriccoli, la justice hégémonique et la justice négociée servent à indiquer en même temps la mutation et les piliers de cette dimension plurielle de la justice. Je m'arrête brièvement sur cette ambivalence.

Sous le premier aspect: la justice négociée opère déjà dès la première phase de l'expérience juridique médiévale; la justice hégémonique au contraire, s'affirme entre le XIII ${ }^{\mathrm{e}}$ et le $\mathrm{XIV}^{\mathrm{e}}$ siècle. Elle côtoie la justice négociée et en prend progressivement la place, constituant la plate-forme sur laquelle sera édifié le pénal à l'époque contemporaine ${ }^{36}$.

À la base de ce long processus, qui, de la crise du modèle communal mène à l'affirmation des régimes seigneuriaux puis, à l'ère moderne, à la réalisation de formations politiques étatiques, avec la transmutation corrélative du système de pouvoir municipal, qui, d'une phase 'communautaire' (où la règle coutumière avait la première place), passe à une phase 'autoritaire' (où commence à compter davantage la règle voulue par le prince) $)^{37}$. Il représente un nouveau rapport entre le droit et le pouvoir dans lequel la Respublica exerce de manière stratégique le droit de punir «pour se protéger $»^{38}$.

\footnotetext{
Sbriccoli (2001, pp. 356-364). Voir aussi ce qu'a observé à ce propos Lacchè (2005, p. 411sqq.). Sbriccoli (2001, pp. 356-359; 2002, pp. 164-167).

34 Sbriccoli (2001, p. 361). Cette justice est en effet «marquée par un caractère prononcé d'apparat, fondée sur la sujétion, dirigée principalement vers la punition du coupable». C'est pour cela qu'elle propose un caractère rituel plus formalisé, qui préfère l'écriture à l'oralité et se fonde «sur quatre présupposés techniques: la loi, l'action, la preuve, la peine» (p. 360). Cf. Sbriccoli (2002, p. 169). Sbriccoli (2001, p. 360; 1998, p. 263; 2002, pp. 169-171; 1991c, p. 21).

Sbriccoli (2004, pp. 95-96).

Sbriccoli (2002, pp. 167, 171-172).

38 Sbriccoli (2003, p. $71 ; 2004$, p. 102).
} 
Mais la justice négociée et la justice hégémonique sont des expressions qui indiquent aussi les piliers du système judiciaire entre le Moyen-Âge et l'époque moderne. En effet, celles-ci, bien que portant en elles-mêmes des conceptions opposées de la justice, tendent, en réalité, à se combiner dans un régime relativement variable, mais fondamentalement stable pendant tout l'Ancien Régime ${ }^{39}$.

Le nouveau registre hégémonique de la justice ne réussira pas à s'affirmer complètement; il se limitera à concerner le haut criminel et il aura la capacité de pénétrer le territoire en laissant à l'écart les périphéries, en conservant des espaces libres, externes au jugement 'public', au profit des pratiques de la justice traditionnelle ${ }^{40}$. Mais il y a plus: la justice négociée «préservera son noyau distinctif en le nichant dans les pratiques qui présideront à la nouvelle manière de rendre la justice, tandis que son esprit continuera à conditionner les systèmes pénaux, jusqu'à la fin des anciens régimes ${ }^{41}$. Il se produit - dit encore Sbriccoli - un phénomène d' «homologation de la justice négociée 'traditionnelle', qui petit à petit finit par prendre les façons, le style de la justice hégémonique - au point d'être absorbée par celle-ci $»^{42}$. Il s'agit d'une perméabilité réciproque par laquelle la justice négociée, en se conformant à la justice hégémonique, finit par conférer à celle-ci certains aspects de négociation qui ne lui appartenaient pas originellement. Pensons à la 'médiation' au moment de l'exécution pénale, qui occupe une place importante dans les systèmes pénaux d'Ancien Régime ${ }^{43}$.

Selon Sbriccoli, la possibilité technique de cette homogénéisation réside dans le rapport entre la dimension procédurale et la dimension substantielle du droit. Aujourd'hui, nous sommes habitués à concevoir le primat du droit pénal substantiel, qui prédéfinit et circonscrit le domaine de d'action du processus répressif; mais dans le pénal de la fin du Moyen-Âge, ce rapport apparaît inversé. Les normes substantielles «ne sont ni prioritaires, ni présupposées, mais laissées au juge dans le cadre de jugements interprétatifs sur les actes des justiciables, dont il faut avant tout évaluer la dépravation (pravitas), la nocivité, et le degré de désobéissance qu'elles expriment». Voilà pourquoi cette justice hégémonique s'expose nécessairement «aux infiltrations du négocié » et rendent «difficile l'émersion d'un procès pénal à son tour autonome ${ }^{44}$.

L'absence de subordination de la dimension procédurale à la dimension substantielle constitue, sur le plan de la technique juridique, une preuve de l'existence de différentes formes de justice que le terrain pluriforme du procès permet justement de faire émerger dans leur équilibre effectif.

39 Sbriccoli (2004, p. 109).

$40 \quad$ Sbriccoli (2004, pp. 109-110). Voir aussi Sbriccoli (1991c, p. 20; 1997a, p. 24; 2001, p. 357; 2002, p. 169).

41 Sbriccoli (2002, p. 167). Cf. Sbriccoli (2003, p. 59).

42 Sbriccoli (2001,p. 363). La persistance d'une dimension négociée de la justice, dans un cadre où prédomine la justice hégémonique, est particulièrement évidente en Italie, où les formations politiques sont destinées à perdre leur autonomie de sorte que le soutien constitutionnel de l'État émergeant vient à manquer à la consolidation du pénal hégémonique; voir Sbriccoli (2004, pp. 116-118).

43 Cf. Sbriccoli (2001 p. 363; 2002, p. 172).

44 Sbriccoli (2002, pp. 177-178). Cf. Meccarelli (2002, pp. 706-707; 2007, p. 580). 


\section{LE «DROIT RITUEL» DANS LA DIMENSION DE LA JUSTICE PLURIELLE}

Revenons donc au problème du rite, afin de mieux évaluer le sens de cette approche au thème du procès pénal.

Mario Sbriccoli observe, en effet, la dimension plurielle de la justice, redécouverte à la lumière de ces catégories organisatrices, sur le terrain plus strictement technico-juridique, par rapport «à l'ensemble complexe d'opérations et de rites judiciaires que l'on trouve au sein des nombreux (et différents) 'procès' et dans toutes les combinaisons spatio-temporelles possibles ${ }^{45}$. À ce propos, il parle de droit rituel vu comme «l'ensemble des règles (ou des pratiques) qui, par rapport aux institutions judiciaires, disciplinent les compétences et le déroulement ${ }^{46}$. Il propose en outre de désarticuler la catégorie 'procès pénal' ${ }^{47}$-, incapable de comprendre dans son ensemble un tel panorama articulé de rites procéduraux - et de la poursuivre à l'intérieur des pratiques; cela justement afin «d'éviter les équivoques, les exclusions ou les anachronismes ${ }^{48}$ et de mettre l'accent sur la justice pénale pratiquée, même quand on étudie le point de vue scientifique-doctrinal du procès.

La perspective qui en découle, dirais-je, est celle d'une histoire de la dimension doctrinale du procès dans une perspective phénoménologique. Elle examine les différentes procédures du point de vue du système en acte, c'est-à-dire en considérant leurs enchaînements possibles et effectifs et les développements évolutifs qui y sont liés, pour reconstruire, du point de vue technico-juridique, le sens historique de cette dimension de justice.

Mario Sbriccoli relit de manière innovatrice les concepts juridiques d'accusatio et d'inquisitio: ils «semblent correspondre à des étapes du processus, ou a ses phases, qui se combinent différemment en fonction de la dynamique propre de chaque cas ${ }^{49}$. Ce qui ne signifie pas ne pas en reconnaître les particularités et les éléments de différenciation, qu'il repère et souligne lui aussi avec précision ${ }^{50}$. C'est un autre point qui nous intéresse ici: reconsidérée de la sorte, la composition entre le registre accusatoire et le registre inquisitoire donne vie à une procédure qui «apparaît mixte, qualifiée de manière croissante par l'expansion de la fonction inquisitoire, conditionnée toutefois par le comportement encore extra-processuel des parties impliquées $»^{51}$.

45 Sbriccoli (1988, p. 498, note 15).

46 Sbriccoli (1988, p. 498); cf. Sbriccoli (2001, p. 355).

47 Sbriccoli (2001, p. 348). En effet, celle-ci n'est, pour les époques qui nous intéressent, entièrement comprise ni par les règles du procès romain canonique, ni par la législation.

48 Sbriccoli (1988, p. 498, note 15).

49 Sbriccoli (1998, pp. 246 et 233sqq.). Cf. Sbriccoli (1991c, p. 20; 1997a, p. 48; 2002, p. 168).

50 Sbriccoli (1998, p. 235) reconnaît trois profils de différenciation de ces régimes procéduraux : la qualification de l'action; la typologie des délits auxquels ils se réfèrent tendanciellement (les violences mineures pour l'accusatoire, le haut et le moyen criminel pour l'inquisitoire); le modèle de référence des sources (l'accusatoire pris par le modèle romanistique conservé par les notarii et par les curiales, l'inquisitoire tiré des pratiques et des textes), «jusqu'à devenir un troisième genre».

51 Sbriccoli $(1998$, p. 246). Le caractère hybride des procédures est confirmé par les pratiques judiciaires. Cf. Zorzi (2005, pp. 11-14); Vallerani (2005, pp. 11-14, passim). 
C'est sur cette ligne que se place aussi une tentative de reconstruction du rapport entre ces «manières procédurales» et les dynamiques de pacification transactionnelles $^{52}$. Il a pu de cette façon, du point de la poursuite, mettre en évidence l'existence, dans la justice plurielle, d'une liaison entre une orientation fortement privatiste et, en sens inverse, une orientation publiciste.

Mais ce ne sont pas seulement les catégories générales du procès qui sont vivifiées par l'histoire de sa dimension doctrinale développée dans une perspective phénoménologique; celle-ci permet en effet aussi de considérer des aspects spécifiques de la procédure sans tomber dans une reconstruction techniciste et décontextualisée. Chaque institution procédurale est plutôt comprise dans sa fonction par rapport aux tendances au changement, qui traversent la dimension de la justice. Une telle position amène à considérer les moyens procéduraux par rapport au type de justice qu'ils arrivent à induire, en les transformant en «indicateurs de la 'nature' du système punitif $»^{53}$. Il en est ainsi par exemple pour certaines catégories repérées comme les pivots technico-juridiques de la transformation du droit rituel en une perspective hégémonique; il suffit de penser à l'action, à la preuve, à la peine ${ }^{54}$.

Cette approche de l'étude du procès judiciaire est d'une utilité certaine sur le terrain sociopolitique et institutionnel, comme l'historiographie du Moyen-Âge la plus avertie l'a prouvé, mais elle est fondamentale aussi du point de vue plus spécifiquement historico-juridique: elle permet de dépister et d'expliquer la phase d'émersion du pénal et certaines prémisses de ce que sera ensuite, au XIX ${ }^{\mathrm{e}}$ siècle, la phase de la construction du pénal ${ }^{55}$.

\section{LA DOCTRINE JURIDIQUE ET LA PHÉNOMÉNOLOGIE DU PROCÈS}

Il y a un troisième aboutissement de l'itinéraire historiographique de Mario Sbriccoli sur lequel je voudrais m'arrêter. Il s'agit du discours sur l'importance de la source doctrinale dans ce cadre de reconstruction phénoménologique.

Pour Sbriccoli, le juriste s'insère dans le rapport qui s'instaure entre les processus de pouvoir et les moyens juridiques. Ils effectuent une médiation entre les intérêts sociaux les plus importants qu'ils pondèrent par le droit ${ }^{56}$. Les juristes, en outre, jouent aussi le rôle de contrepoids constitutionnel ${ }^{57}$ dans un horizon comme celui de l'époque médiévale finissante et de l'Ancien Régime, dans lequel les équilibres du pouvoir tirent du moment politique leurs poussées dynamiques, mais non pas leur niveau d'ordre.

La dimension de la justice criminelle est un terrain utile de vérification, dans le concret, de la fonction du juriste, car c'est aussi le terrain sur lequel se joue la redéfinition des nouveaux rapports de pouvoir et du rapport entre le pouvoir et le droit.

52 Sbriccoli (1998,pp. 233-240) : le recours au procès accusatoire (mais ce discours serait valable aussi pour l'inquisitoire) devient le «moyen pour obliger le coupable à accepter une transaction». Cf. Sbriccoli (2002, p. 165).

53 Sbriccoli (1998, p. 232).

54 Cf. Sbriccoli (1991c, p. 21; 1998, pp. 232-233, 246-249; 2001, p. 360; 2002, pp. 168-171).

55 Sbriccoli (1988, pp. 498-500).

56 Comme indiqué supra (note 2), c'est un aspect que l'on retrouve constamment dans la production scientifique de Mario Sbriccoli, à partir de sa première monographie. Cf. Grossi (2007, pp. 457-458).

57 Sbriccoli (1991c, p. 31). 
La doctrine est redécouverte dans sa capacité à s'acquitter de son rôle consistant à adapter l'ordre juridique aux nouveaux équilibres constitutionnels, qui mûrissent au fur et à mesure que s'achèvent les autonomies communales et que naissent les États juridictionnels ${ }^{58}$ : «l'objectif politique de la justice s'entrecroise avec la doctrine des juristes. Ils lui confèrent la rationalité, les bases techniques, la crédibilité et l'approbation, outre l'indispensable confirmation de sa légitimité».

Dans ce cas aussi des réponses précises jaillissent des conclusions auxquelles parvient Mario Sbriccoli.

Un premier exemple important est constitué par la détermination du rôle décisif du développement doctrinal dans la rationalisation de l'entrelacement, dialectique et en même temps coopératif, qui s'est produit au niveau de la justice pratiquée, entre accusatio et inquisitio ${ }^{59}$.

Le même discours vaut pour l'apport de la doctrine à la construction de la silhouette juridique qui rend typique et stabilise la justice hégémonique. Ce sont les juristes, avec leurs œuvres doctrinales, qui intègrent les critères typiques de la justice hégémonique dans la justice pénale ordinaire ${ }^{60}$. Techniquement, cette opération, s'est manifestée à travers la généralisation à tous les types de délits du paradigme $d u$ délit politique entendu comme principe d'obéissance, en traduisant en langage scientifique (et donc en stabilisant dans le temps) ce qui se produisait dans la pratique $^{61}$.

Dans l'opération de synthèse doctrinale consacrée aux questions de nature procédurale, qui naît du vidi communiter observari, le juriste de ius commune ajoute des valeurs et des contenus qui sont, si l'on peut dire, appropriés à la réalité décrite, mais pas propres à cette réalité, originellement. En particulier par cette doctrine, il a été question - les recherches de Mario Sbriccoli l'ont bien mis en évidence - de dégager le format pénal des conflits, et de mettre en œuvre avec celle-ci une première base de concepts et de catégories afin de construire le droit pénal substantiel ${ }^{62}$.

58 Sbriccoli (2002, pp. 175, 173-178; 2004, p. 102); à ce propos, le genre doctrinal de la practica criminalis est particulièrement efficace. Cf. Sbriccoli (1986b, p. 488).

59 Sbriccoli,(1998,pp. 235-236). En particulier, l'on reconnaît l'œuvre constructive de la doctrine dans le fait de faire émerger «de l'intérieur d'un intérêt privé et particulier [...] un profil immatériel (que nous pourrions nommer aujourd'hui symbolique) qui constitue la base du publice interest et du devoir public - l'officium - qui en découle. La violation de droit se sépare de la lésion du bien et le bien lésé se divise en deux logiques distinctes qui donnent naissance à deux aspects différents: le matériel, concrètement appréciable, qui appartient à la victime, et qui doit être dédommagé ; l'immatériel, politique, qui appartient à la communitas - ou à la civitas - et qui doit être rétribué par la peine. Comme on le voit, une parfaite abstraction. Ceci advient chaque fois que le juridique effectue un saut de qualité, ou se situe à un tournant dans l'histoire de sa progression sur la voie de l'adhésion aux nouvelles exigences de la société qui augmentent en se compliquant. Une abstraction résout un problème politique né de la complexité, et le fait en "constituant" la base, c'est-à-dire la supposition, de la solution, de manière telle qu'elle - la solution - ne pourra être que juridique» (p. 267). Cf. Sbriccoli (2002, p. 175).

Sbriccoli (2001, p. 363; 1991c, p. 18).

61 Sbriccoli $(2004$, p. 102, note 30; 2001, p. 362; 1998, p. 263). Il avait considéré le système des délits politiques comme speculum societatis déjà dans Sbriccoli (1974, pp. 4-5, passim). Cf. Grossi (20042005, pp. 1394-1395); Lacchè (2005, p. 405sqq.).

62 Voir par exemple Sbriccoli (2002, pp. 173-178). C'est une opération, explique-t-il (Sbriccoli, 2004, p. 104), que Alberto da Gandino avait déjà initiée précocement; mais c'est la pensée de Deciani qui représentera le moment majeur de maturation de cette orientation programmatique de la doctrine: «forte d'une plus grande conscience, elle fait du format pénal du conflit, le point de départ d'une nouvelle théorie (qui fonde le rôle de la loi) et l'axe portant de l'autonomie du ius criminale». Cf. Sbriccoli (1988, pp. 498-499). 
Il s'est agi d'un travail fondamental pour inspirer les contenus des principales législations criminelles, qui, à l'époque moderne, émaneront des Princes, et cela toujours dans une optique de consolidation de (leur) justice hégémonique ${ }^{63}$.

De la sorte, la doctrine décrit la pratique pénale, produisant aussi bien un discours constitutif de l'appareil de la haute justice qu'un discours réflexif de la justice plurielle ${ }^{64}$, en ce sens que, «tandis qu'elle fonde et explique les engrenages et les raisons d'un système punitif, elle rend également largement compte des contextes qui lui sont corrélés ${ }^{65}$.

Pour Sbriccoli, cela ne veut pas dire que toute la multiplicité des niveaux dans lesquels la dimension de la justice se manifeste concrètement sont envisagés par la lecture doctrinale ${ }^{66}$. Toutefois, dans ce développement scientifique du problème de la justice, l'on fait entrer en ligne de compte les contextes et les tendances en acte et l'on explique quels sont les instruments stratégiques afin de les satisfaire ou de les gouverner.

Si j'interprète correctement sa pensée, je peux dire que nous pouvons considérer la dimension doctrinale du procès comme un point efficace d'observation unitaire (car compréhensif, bien que d'une façon non exhaustive) de la justice plurielle.

\section{LES PERSPECTIVES HISTORIOGRAPHIQUES}

Je passe donc à la formulation de quelques réflexions d'ensemble sur les perspectives historiographiques. Il me semble que l'on peut, voire que l'on doive, continuer d'interroger les sources doctrinales du procès d'un point de vue qui tienne compte de la 'disposition à l'effectivité' de cette pensée juridique.

Il est certain que de nombreux aspects de la phase d'émersion du pénal et de l'histoire du procès qui l'encadre ont été éclaircis. Il y a encore toutefois des terrains à défricher.

Je pense tout particulièrement, en tant qu'historien du droit, aux catégories systématiques qui soutiennent le jeu créatif d'où naît le pénal, entre les pratiques de justice et ses lectures doctrinales. Si je me réfère à mes expériences de recherche, les deux éléments conceptuels ordinarium/extraordinarium en représentent un exemple ${ }^{67}$.

${ }_{63}$ Cf. Sbriccoli (2001, pp. 347-348; 2002, p. 181; 2003, pp. 79-88; 2004, passim et en particulier pp. 98-99, 104-105, 118-119; 2007).

${ }_{64}$ Sbriccoli (2001, p. 347).

${ }_{65}$ Sbriccoli (1988, p. 501). Cf. Sbriccoli (1991d, p. 11; 1986a, pp. 141-145).

66 Sbriccoli (2001, p. 348). Sur le plan méthodologique, il ne faut pas oublier qu'afin d'avoir une perception complète de la dimension justice, il faut toujours intégrer la source doctrinale avec les sources d'archives, et avant tout les archives judiciaires. Voir aussi Sbriccoli (1988, p. 494; 1986a, p. 131).

${ }^{67}$ Sur la base de cette distinction, la complexité de procédure est décrite par la doctrine sur un plan à deux dimensions de sorte que l'extraordinarium représente le niveau de modernisation et d'adéquation permanente de l'ordinarium. Du côté de l'aspect extraordinaire l'on expérimente, en effet, des modalités de solution des conflits qui tendent elles-mêmes à devenir ordinaires. La génération de la manière procédurale inquisitoire s'explique bien, par exemple, à travers ces catégories. Sur ce point voir Meccarelli (1998, pp. 288-298, 367-376; 2007, pp. 575-578). Pour une observation de cette dynamique du point de vue des pratiques judiciaires et une réflexion sur les connexions de ses valeurs par rapport à la dialectique politique dans les villes, voir la reconstruction faite sur Bologne entre le XIII ${ }^{\mathrm{e}}$ siècle et le XIV ${ }^{\mathrm{e}}$ siècle par Vallerani (2005, pp. 16-17, 247-270). 
À travers ces catégories théoriques, la complexité procédurale de la justice pratiquée est décrite (et conçue) sur le plan doctrinal comme 'système'. Et cela sans devoir recourir à des mécanismes systématiques de fermeture autoréférentielle ${ }^{68}$.

Une recherche sur le dimensionnement doctrinal de la flexibilité procédurale, peut être considérée au moins sous quatre aspects ${ }^{69}$ : la variabilité du déroulement; le caractère 'ouvert' de la clôture du procès; l'accidentalité du sujet de droit; les régimes de la juridiction (concurrence, exclusion, articulation).

Sous le premier aspect, il serait par exemple intéressant d'analyser de plus près quelles sont les catégories théoriques pensées pour régler les régimes d'enchaînement variable des modules procéduraux hétérogènes. Cela aussi dans la perspective de reconstruire le rapport entre les catégories ordinatrices du procès et les catégories générales du système juridique. Sur ce terrain par exemple, l'on pourrait considérer l'apport spécifique de la doctrine de droit canonique à la production des concepts et des institutions capables de favoriser une caractérisation flexible du rite; je pense par exemple aux soi-disant clausulae diminuentes iuris ordinem ${ }^{70}$. Mais il me semble que l'on pourrait mener une recherche avec la même méthode aussi sur les terrains de la preuve et de la peine.

Un autre versant que l'on peut analyser - bien qu'il dépasserait la chronologie de la première époque moderne - est celui de la genèse des modèles procéduraux. L'objet de la recherche serait la signification, sur le plan des idéologies, de la standardisation du rite procédural, ou mieux de la cristallisation des «manières procédurales » en modèles procéduraux.

Venons-en maintenant au «caractère 'ouvert' du moment de la clôture du procès »; les objets de la recherche seraient, dans ce cas, les institutions qui, sur le plan doctrinal, en suivant la logique de cette justice plurielle, peuvent faire office d'instruments pour régler (rebus sic stantibus) la conclusion du rite et de façon plus générale les modes de sortie des procès ${ }^{71}$. Je pense en ce moment aux régimes juridiques complexes de la absolutio (par exemple la soi-disant absolutio cum clausula ou ab instantia ou bien cum cautione, cum fideiussione, etc.), ou à ceux de la transactio, qui tendent à faire osciller l'affaire judiciaire entre le registre hégémonique et celui de la négociation, en interrompant, renvoyant, déplaçant le procès. Je ne négligerai pas non plus de considérer le versant de la condamnation. Ici aussi il reste un caractère ouvert au moment de la clôture du procès. Que l'on pense aux négociations sur l'exécution de la peine ou à la révision de la condamnation.

Puis un troisième aspect est celui que j'ai défini comme l' ''accidentalité' du sujet de droit». Il se réfère aux acteurs qui entrent dans le jeu procédural soit qu'ils

68 Je me réfère au type de clôture systématique - présent, non pas par hasard, dans les systèmes basés sur le principe de la légalité - qui se produit quand la dimension factuelle à laquelle s'applique le droit devient le simple aboutissement d'un discours juridique déjà accompli à l'intérieur des dispositions normatives prévues. Schütz (1995, pp. 113-123), a indiqué comment une «politique constructive de la réponse à la transgression » est réalisée dans ces systèmes. Vice versa dans le ius commune se produit une «politique fictive de l'autodéfense du droit»: la norme juridique maintient sa vigueur dans le temps, justement parce qu'on peut en suspendre l'application lorsqu'elle résulte inadéquate. Cf. Meccarelli 1998, pp. 318-325; 2007, p. 577).

Je reprends ici la classification proposée dans Meccarelli (2007, pp. 578-582).

70 Cf. Meccarelli (1998, pp. 255-306); Alonso Romero (2001, pp. 40-53).

71 Pour les implications de ce point de vue de l'étude du procès dans une dimension de l'histoire politique de la justice voir Vallerani (2005, p. 16, passim). 
sont légitimes, soit qu'ils sont impliqués dans celui-ci à un titre quelconque ${ }^{72}$. Nous avons de nombreux témoignages sur ce point dans les sources d'archives; il serait intéressant de mener une recherche visant les catégories doctrinales qui traduisent sur le plan du savoir (et donc du système) cette dynamique de la justice pratiquée.

Il reste enfin le quatrième aspect: celui des «régimes de la juridiction (concurrence, exclusion, articulation)». Il s'agit dans ce cas de considérer les instruments juridiques aptes à régler les régimes des déplacements de juridiction, qui accompagnent souvent (ou sont à la base de) la variabilité des parcours procéduraux à laquelle nous avons plusieurs fois fait allusion. Ce point revêt une grande importance dans les systèmes comme ceux de l'époque médiévale et de l'époque moderne caractérisés par un pluralisme juridictionnel important ${ }^{73}$ et par la variabilité des équilibres concrets entre les pouvoirs politiques ${ }^{74}$. Nous pouvons donc dire que la juridiction pénale n'est pas répartie, mais partagée entre des juridictions concurrentes et que la justice administrée est le résultat de l'équilibre effectif qu'elles assument réciproquement à chaque fois. À ce propos, nous pourrions examiner les critères mis en place par les juristes, pour combiner, sur le terrain du 'droit rituel', la coexistence de juridictions concurrentes, prises comme élément organique de la culture judiciaire. Ceux-ci pourraient non seulement se révéler comme pouvant être référés à un cadre d'ensemble, mais ils pourraient aussi éclaircir de quelle façon et dans quelle mesure la justice devient le terrain du développement de la confrontation politique, qui se déroule sur le terrain de la iurisdictio.

Les domaines de recherche que j'ai illustrés ici ont tous un caractère historicojuridique très prononcé. Ils ont toutefois une implication directe dans la justice pratiquée. S'ils étaient 'intégrés' dans une perspective d'histoire de la justice criminelle, ils seraient très utiles pour comprendre non seulement l'histoire de la justice, mais aussi les dynamiques concrètes de légitimation et de construction de l'espace public dans les villes médiévales ${ }^{75}$ et dans les systèmes de l'Ancien Régime.

Ébauchées, mes remarques ne constituent pas un programme précis de recherche. Elles veulent simplement témoigner sur l'actualité méthodologique et historiographique de Mario Sbriccoli dont la leçon par sa pertinence constitue pour longtemps une référence importante dans le parcours que doivent accomplir les juristes et les historiens.

\author{
Massimo Meccarelli \\ Università di Macerata \\ Istituto di studi storici \\ Via Garibaldi, 20 \\ I - 62100 MACERATA \\ meccarelli@unimc.it
}

72 C'est-à-dire que le système de procédure se ressent, d'une manière beaucoup plus accentuée, par rapport à notre façon de concevoir la justice criminelle, de l'importance collective du fait judiciaire. Avant d'être un instrument de sauvegarde ou de punition d'un individu, celui-ci est un instrument de recherche de paix sociale, de définition des équilibres entre les corps sociaux; son articulation concrète trouve sa place, en somme, à l'intérieur de la dialectique politique entre les pouvoirs qui se reflètent forcément sur l'affaire juridique spécifique. La justice médiévale s'occupe de conflits qui, avant de représenter « une déviance ou une pathologie sociale» sont «un mode de relation ordinaire, comme un processus social ouvert», explique clairement A. Zorzi (1996, p. 21); cela, pour le dire avec Vallerani (2005, p. 13), fait que le procès devient «un micro événement social».

73 Cf. Grossi (1995, passim et en particulier pp. 29-35, 130-135, 223-235); Chittolini (1994, pp. 582586); Costa (1999, pp. 9-19); Mannori, Sordi (2001, pp. 17-20).

74 Zorzi (2005, pp. 13-34).

75 Une confirmation en ce sens est donnée dans les pages récentes de Zorzi (2007, pp. 173-178). 


\section{BIBLIOGRAPHIE}

Alonso Romero, M.P., El solemne orden de los juicios. La lenitud como problema en la historia del proceso en Castilla, Anuario de la Facultad de derecho de la Universidad autónoma de Madrid, 2001, 5, pp. 23-53.

Cappellini, P., Mario Sbriccoli, il giurista civile, in Lacchè, L., Latini, C., Marchetti, P., Meccarelli, M., (dir.), Penale, Giustizia, Potere. Metodi, Ricerche, Storiografie. Per ricordare Mario Sbriccoli, Macerata, EUM, 2007, pp. 59-106.

Caroni, P., Blicke über den Gartenzaun. Von der Beziehung der Rechtsgeschichte zu ihren historischen Nachbarwissenschaften, in Pahlow, L. (dir.), Die zeitliche Dimension des Rechts. Historische Rechtsforschung und geschichtliche Rechtswissenschaft, PaderbornMünchen-Wien-Zürich, Schöning, 2005, pp. 27-55.

Chittolini, G., Il 'privato', il 'pubblico', lo Stato, in Chittolini, G., Mohlo, A., Schiera, P., (dir.), Origini dello Stato. Processi di formazione statale in Italia fra medioevo ed età moderna, Bologna, il Mulino, 1994, pp. 553-589.

Costa, P., Civitas storia della cittadinanza in Europa, 1. Dalla civiltà comunale al Settecento, Roma-Bari, Laterza, 1999.

Costa, P., Politica, diritto, società: la 'storiografia civile' di Mario Sbriccoli, in Lacchè, L., Latini, C., Marchetti, P., Meccarelli, M., (dir.), Penale, Giustizia, Potere. Metodi, Ricerche, Storiografie. Per ricordare Mario Sbriccoli, Macerata, EUM, 2007, pp. 41-57.

Grossi P. (dir.), Storia sociale e dimensione giuridica. Strumenti di indagine e ipotesi di lavoro, Milano, Giuffrè, 1986.

Grossi, P., L'ordine giuridico medievale, Roma-Bari, Laterza, 1995.

Grossi, P., Ricordo di Mario Sbriccoli, Quaderni fiorentini per una storia del pensiero giuridico moderno, 2004-2005, 33-34, pp. 1391-1399.

Grossi, P., Conclusioni, in Lacchè, L., Latini, C., Marchetti, P., Meccarelli, M., (dir.), Penale, Giustizia, Potere. Metodi, Ricerche, Storiografie. Per ricordare Mario Sbriccoli, Macerata, EUM, 2007, pp. 453-459.

Lacchè, L., Mario Sbriccoli (1941-2005), Rivista di storia del diritto italiano, 2005, LXXVIII, pp. 407-420.

Lacchè, L., Introduzione in Lacchè, L., Latini, C., Marchetti, P., Meccarelli, M., (dir.), Penale, Giustizia, Potere. Metodi, Ricerche, Storiografie. Per ricordare Mario Sbriccoli, Macerata, EUM, 2007, pp. 9-35.

Lacchè, L., Latini, C., Marchetti, P., Meccarelli, M., (dir.), Penale, Giustizia, Potere. Metodi, Ricerche, Storiografie. Per ricordare Mario Sbriccoli, Macerata, EUM, 2007.

Madero, M., Conte, E., (dir.), Procesos, inquisiciones, pruebas. Homenaje a Mario Sbriccoli (1941-2005), Buenos Aires, Universidad Nacional de General Sarmiento (à paraître).

Mannori, L., Sordi, B., Storia del diritto amministrativo, Roma-Bari, Laterza, 2001.

Meccarelli, M., Arbitrium. Un aspetto sistematico degli ordinamenti giuridici in età di diritto comune, Milano, Giuffrè, 1998.

Meccarelli, M., Tortura e processo nei sistemi giuridici dei territori della Chiesa. Il punto di vista dottrinale (secolo XVI), in Durand, B. (dir.), La torture judiciaire. Approches historiques et juridiques, Lille, Centre d'histoire judiciaire, 2002, vol. II, pp. 677-707.

Meccarelli, M., Le categorie dottrinali della procedura e l'effettività della giustizia penale nel tardo Medioevo, in Chiffoleau, J., Gauvard, C., Zorzi, A., (dir.), Pratiques sociales et politiques judiciaires dans les villes de l'Occident à la fin du Moyen-Âge, Roma, École française de Rome, 2007, pp. 573-594.

Sbriccoli, M., L'interpretazione dello Statuto. Contributo allo studio della funzione dei giuristi nell'età comunale, Milano, Giuffrè, 1969. 
Sbriccoli, M., Politique et interprétation juridique dans les villes italiennes du Moyen-Âge, Archives de Philosophie du Droit, 1972, XVII, pp. 99-113.

Sbriccoli, M., Crimen lease maiestatis. Il problema del reato politico alle soglie della scienza penalistica moderna, Milano, Giuffrè, 1974.

Sbriccoli, M., Recensione a Materiali per una storia della cultura giuridica, V, 1975, La questione criminale, 1976, II, pp. 205-210.

Sbriccoli, M., Storia del diritto e storia della società. Questioni di metodo e problemi di ricerca, in Grossi, P. (dir.), Storia sociale e dimensione giuridica. Strumenti di indagine e ipotesi di lavoro, Milano, Giuffrè, 1986a, pp. 127-148.

Sbriccoli, M., Brigantaggio e ribellismi nella criminalistica dei secoli XVI-XVIII, in Ortalli, G. (dir.), Bande armate, banditi, banditismo e repressione di giustizia negli Stati europei di antico regime, Roma, Jouvence, 1986b, pp. 479-500.

Sbriccoli, M., Fonti giudiziarie e fonti giuridiche. Riflessioni sulla fase attuale degli studi di storia del crimine e della giustizia criminale, Studi storici, 1988, XXIX, 2, pp. 491-501.

Sbriccoli, M., Histoire de la criminalité et histoire pénale. Le problème des sources juridiques dans l'histoire du crime et de la justice criminelle, International Association for the History of Crime and Criminal Justice Bulletin, 1991a, 14, pp. 86-102.

Sbriccoli, M., Le droit pénal, le crime et la justice criminelle ont une histoire, Déviance et Société, 1991b, 15, 4, pp. 377-379.

Sbriccoli, M., «Tormentum idest torquere mentem». Processo inquisitorio e interrogatorio per tortura nell'Italia comunale, in Maire-Vigueur, J.-C., Paravicini Bagiani, C. (dir.), La parola all'accusato, Palermo, Sellerio, 1991c, pp. 17-32.

Sbriccoli, M., Nox quia nocet. I giuristi, l'ordine e la normalizzazione dell'immaginario, in Sbriccoli, M. (dir.), La notte. Ordine, sicurezza e disciplinamento in età moderna, Firenze, Ponte alle Grazie, 1991d, pp. 9-19.

Sbriccoli, M., «Storia del diritto italiano»: articolazioni disciplinari vecchie e nuove, in Grossi, P. (dir.), L'insegnamento della storia del diritto medievale e moderno: strumenti, destinatari, prospettive, Milano, Giuffrè, 1993, pp. 117-133.

Sbriccoli, M., Legislation, Justice and Political Power in Italian Cities, 1200-1400, in Padoa Schioppa, A. (dir.), Legislation and Justice: The Origins of the Modern State, $13^{\text {th }}-18^{\text {th }}$ Centuries, Oxford, Oxford University Press, 1997a, pp. 37-55.

Sbriccoli, M., Gobernar castigando. Francisco Tomás y Valiente e la storia del diritto penale moderno, Quaderni fiorentini per la storia del pensiero giuridico moderno, 26, 1997b, pp. 689-715.

Sbriccoli, M., «Vidi communiter observari». L'emersione di un ordine penale pubblico nelle città italiane del secolo XIII, Quaderni fiorentini per la storia del pensiero giuridico moderno, 1998, 27, pp. 231-268.

Sbriccoli, M., Giustizia negoziata, giustizia egemonica. Riflessioni su una nuova fase degli studi di storia della giustizia criminale, in Bellabarba, M., Schwerhoff, G., Zorzi, A., (dir.), Criminalità e giustizia in Germania e in Italia. Pratiche giudiziarie e linguaggi giuridici tra tardo medioevo ed età moderna, Bologna, il Mulino / Berlin, Duncker u. Humblot, 2001, pp. 345-364.

Sbriccoli, M., Giustizia criminale, in Fioravanti, M. (dir.), Lo Stato moderno in Europa. Istituzioni e diritto, Roma-Bari, Laterza, 2002, pp. 163-205.

Sbriccoli, M., La benda della giustizia. Iconografia, diritto e leggi penali dal medioevo all'età moderna, in Sbriccoli, M., Costa, P., Fioravanti, M. et al., Ordo iuris. Storia e forme dell'esperienza giuridica, Milano, Giuffrè, 2003, pp. 41-95.

Sbriccoli, M., Lex facit delictum. Tiberio Deciani e la criminalistica italiana nella fase cinquecentesca del penale egemonico, in Cavina, M. (dir.), Tiberio Deciani (1509-1582). Alle origini del pensiero giuridico moderno, Udine, Forum, 2004, pp. 91-119. 
Sbriccoli, M., Histoire sociale et dimension juridique: l'historiographie italienne récente du crime et de la justice criminelle, Crime, Histoire et Sociétés / Crime, History and Societies, 2007, 11, 2, pp. 139-148.

Schütz, A., L'immaculée conception de l'interprète et l'émergence du système juridique: à propos de «fictions » et «construction» en droit, Droits. Revue française de théorie juridique, 1995, 21, pp. 113-126.

Vallerani, M., La giustizia pubblica medievale, Bologna, il Mulino, 2005.

Zorzi, A., Conflits et pratiques infrajudiciaires dans les formations politiques italiennes du $\mathrm{XIII}^{\mathrm{e}}$ au $\mathrm{XV}^{\mathrm{e}}$ siècle, in Garnot, B. (dir.), L'infrajudiciaire du Moyen-Âge à l'époque contemporaine, Dijon, EUD, 1996, pp. 20-36.

Zorzi, A., Negoziazione penale, legittimazione giuridica e poteri urbani nell'Italia comunale, in Bellabarba, M., Schwerhoff, G., Zorzi, A. (dir.), Criminalità e giustizia in Germania e in Italia. Pratiche giudiziarie e linguaggi giuridici tra tardo medioevo ed età moderna, Bologna, il Mulino / Berlin, Duncker u. Humblot, 2001, pp. 13-34.

Zorzi, A., L'egemonia del penale in Mario Sbriccoli, in Lacchè, L., Latini, C., Marchetti, P., Meccarelli, M., (Dir.), Penale, Giustizia, Potere. Metodi, Ricerche, Storiografie. Per ricordare Mario Sbriccoli, Macerata, EUM, 2007, pp. 155-178. 